\title{
Degenerating Black Saturns
}

\section{Michat Eckstein}

Faculty of Mathematics and Computer Science, Jagiellonian University, ul. Eojasiewicza 6, 30-348 Kraków, Poland

E-mail: michal.eckstein@uj.edu.pl

ABSTRACT: We investigate the possibility of constructing degenerate Black Saturns in the family of solutions of Elvang-Figueras. We demonstrate that such solutions suffer from naked singularities.

KEYwords: Black Holes, Spacetime Singularities

ARXiv EPRINT: 1309.4414 


\section{Contents}

1 Introduction 1

2 Analysis of the limits 2

$2.1 a_{1} \nearrow a_{5}$

$2.2 a_{5} \nearrow a_{4}$

$2.3 a_{3} \nearrow a_{2}$

$2.4 a_{1} \nearrow a_{5} \nearrow a_{4}$

$2.5 a_{1} \nearrow a_{5}, a_{3} \nearrow a_{2}$

$2.6 a_{5} \nearrow a_{4}, a_{3} \nearrow a_{2}$

$2.7 a_{1} \nearrow a_{5} \nearrow a_{4}, a_{3} \nearrow a_{2}$

3 Conclusions $\quad 8$

A The Black Saturn metric $\quad 9$

\section{Introduction}

In [1] Elvang and Figueras have presented a family of axisymmetric black hole solutions to vacuum 4+1-dimensional Einstein equations. Due to the specific topology of the event horizon: $\left.\mathbb{R} \times\left(\left(S^{1} \times S^{2}\right) \cup S^{3}\right)\right)$ it has been named Black Saturn. It can be regarded as a spherical Myers-Perry black hole [2] surrounded by a black ring [3, 4]. The configuration is kept in balance by the angular momenta.

The Black Saturn metrics are of great significance since they provide an example of well-behaved stationary black hole space-times with disconnected Killing horizon. This shows a sharp contrast between solutions to Einstein equations in $4+1$ and $3+1$-dimensions since, as proven recently [5-8], in the latter case analytic stationary two black hole spacetimes are nakedly singular.

The family of Black Saturn solutions is constructed via the inverse scattering method [9] which introduces 8 real parameters: $a_{i}$ with $i=1, \ldots, 5$ and $c_{1}, c_{2}, k$. Moreover, a ninth one $-q$ is brought in by a change of coordinates to facilitate the asymptotic flatness (see [1, p. 10, footnote 3]). The ordering of parameters $a_{i}$ assumed in [1] reads

$$
a_{1} \leq a_{5} \leq a_{4} \leq a_{3} \leq a_{2}
$$

or in terms of the dimensionless ones $\kappa_{i}=\frac{a_{i+2}-a_{1}}{\left(a_{2}-a_{1}\right)^{2}}$,

$$
0 \leq \kappa_{3} \leq \kappa_{2} \leq \kappa_{1} \leq 1 .
$$


A thorough analysis (see $[1,10,11])$ has shown that, under the assumption of parameters $a_{i}$ being pairwise distinct, the metrics in the Black Saturn family describe asymptotically flat, stably causal black hole space-times with smooth domains of outer communications. To guarantee the above listed desired properties of a well-behaved black hole space-time one needs to tune the parameters $c_{1}, c_{2}, k$ and $q$ in terms of $a_{i}$ 's.

The purpose of this work is to investigate the possibility of obtaining a well-behaved metric from the Black Saturn family in the case of coalescence of some of the parameters $a_{i}$. Such coalescence corresponds to the "pole fusion effect" in the inverse scattering method, which may lead to extremal black-hole solutions (see [9, chapter 8.3]). There are however various ways (paths in the parameter space) in which one can obtain a coalescence of two or more $a_{i}$ 's. The result will a priori depend on the chosen limiting procedure as demonstrated in [12]. ${ }^{1}$ For instance, in [1, section A.1] it has been shown that to obtain the limiting case of a Myers-Perry black hole from the balanced Black Saturn configuration one needs to take first $a_{5} \nearrow a_{4}$ and then $a_{1} \nearrow a_{4}$. In general, the assumption that the coalescence is to be considered after the fine tuning of parameters $c_{1}, c_{2}, k, q$ already imposes restrictions on the limiting procedure, since, for example, $a_{1} \nearrow a_{5}$ causes $c_{1}$ to diverge (see $[1,(3.7)]$ or $[10,(2.3)]$ ). Let us note that the parameters $c_{1}$ and $c_{2}$ may $a$ priori assume infinite values. Indeed, the line element (A.1) has a well-defined limit for $c_{1} \rightarrow \pm \infty$ and/or $c_{2} \rightarrow \pm \infty$, which moreover commutes with every coalescence considered in this paper. However, these cases need separate analysis of possible balance conditions.

When the balance conditions are imposed on the Black Saturn solution, the areas of the horizons of the two disconnected components $[1,(3.26),(3.27)]$ tend to zero in the limits $a_{3} \nearrow a_{2}$ and $a_{5} \nearrow a_{4}$ respectively. This suggests that the possible degenerate solutions are nakedly-singular. However, there is no a priori reason for the procedures of coalescence of parameters and imposition of the balance conditions to commute, so this observation does not exclude the possibility of obtaining well-behaved extremal solutions via some other limiting procedure. Let us note, that the same coalescence that leads to the vanishing of the horizon area of the black ring component implies the divergence of its temperature [1, (3.28)]. This suggests that the limiting procedures adopted in [1] are not the right ones, as one should expect $T=0$ for an extremal black-hole solution, since the temperature is proportional to surface gravity.

The strategy we adopt in this paper is to consider the limits $a_{i} \rightarrow a_{j}$ at the level of metric functions of the full Black Saturn solution and then investigate whether the balance conditions can be fulfilled by a fine tuning of parameters $c_{1}, c_{2}, k$ and $q$. To make the paper self-contained we present in the appendix the Black Saturn metric of [1] in generalised Weyl coordinates. For the details of construction and properties we refer the reader to $[1]$ and $[10,11]$.

\section{Analysis of the limits}

Since we are interested only in the solutions with two disconnected components of the event horizon (compare with the rod structure [1, figure 1]), we shall assume the strict

\footnotetext{
${ }^{1}$ We thank Sebastian Szybka for pointing out this reference to us.
} 
inequality $a_{4}<a_{3}$ in the ordering (1.1). We have thus 3 possible two-fold coalescences to be considered in the next subsections. Moreover, there are 3 three-fold and 1 four-fold limit that need to be investigated. When more then two $a_{i}$ parameters coalesce, one can consider various different paths in the parameter space that lead to the same coalescence. Fortunately, if the limiting procedure is performed at the level of the metric, the ordering of the limits does not play a role (compare [1, section A.1]). This is because $a_{i} \nearrow a_{j}$ implies $\mu_{i} \nearrow \mu_{j}$ (A.2) and all of the metric functions (see appendix) are smooth as functions of $\mu_{i}$ 's.

In each of the subsections we consider a particular coalescence of the $a_{i}$ 's parameters while keeping the other distinct. The reason for that is that the behaviour of the metric functions on the axis $(\rho=0)$ should be studied separately in each region of the axis $a_{i} \leq z \leq a_{j}$ (see [10, section 5.4]). This means that each coalescence needs a separate procedure of investigation of the metric functions on the axis.

The detailed analysis of the regularity, asymptotic flatness and causality of the seven limiting cases of the Black Saturn solution is straightforward, but lengthy — one essentially follows the strategy adopted in [10]. However, since our analysis shows that in neither of the investigated limits can one tune the parameters to obtain a balanced configuration we shall only present the part of reasoning that leads to this conclusion.

\section{$2.1 \quad a_{1} \nearrow a_{5}$}

Let us note first, that if one takes the limit $a_{1} \nearrow a_{5}$, then the resulting metric does not depend on the parameter $c_{1}$ anymore. Indeed, $\mu_{1}=\mu_{5}$ implies $M_{1}=M_{3}=M_{4}=0$ (see appendix), thus the parameter $c_{1}$ completely drops out of the line element. According to $[1$, p. 7$]$ this configuration would describe a static black ring around an $S^{3}$ black hole, which are kept apart by a conically singular membrane. Indeed, one can detect the conical singularity by investigating the periodicity of the variable $\varphi$ (compare [10, section 4]). To avoid conical singularities at zeros of the Killing vector $\partial_{\varphi}$ one needs the ratio

$$
\lim _{\rho \rightarrow 0} \frac{\rho^{2} g_{\rho \rho}}{g_{\varphi \varphi}}
$$

to be constant on the set $\left\{z<a_{1}\right\} \cup\left\{a_{4}<z<a_{3}\right\}$, which is an axis of rotation for $\partial_{\varphi}$. By investigating the leading behaviour in $\rho$ of the metric functions $g_{\varphi \varphi}$ and $g_{\rho \rho}$ in the relevant region of the space-time we obtain

$$
\lim _{\rho \rightarrow 0} \frac{\rho^{2} g_{\rho \rho}}{g_{\varphi \varphi}}= \begin{cases}k^{2}, & \text { for } z<a_{1} \\ k^{2} \frac{\left(a_{2}-a_{1}\right)\left(a_{3}-a_{4}\right)^{2}}{\left(a_{3}-a_{1}\right)^{2}\left(a_{2}-a_{4}\right)}, & \text { for } a_{4}<z<a_{3} .\end{cases}
$$

Hence, to avoid conical singularities one would need to have

$$
\frac{\left(a_{2}-a_{1}\right)\left(a_{4}-a_{3}\right)^{2}}{\left(a_{3}-a_{1}\right)^{2}\left(a_{2}-a_{4}\right)}=1,
$$

which is equivalent to

$$
a_{4}=a_{1} \quad \text { or } \quad a_{4}=\frac{a_{1} a_{2}-2 a_{2} a_{3}+a_{3}^{2}}{a_{1}-a_{2}} .
$$


The first case is excluded, whereas the second one would require

$$
\frac{a_{1} a_{2}-2 a_{2} a_{3}+a_{3}^{2}}{a_{1}-a_{2}}<a_{3},
$$

as $a_{4}<a_{3}$ by assumption. The latter however imply that either $a_{3}<a_{1}$ or $a_{3}>a_{2}$, which contradicts the ordering (1.1).

This means that the conical singularity on the axis cannot be avoided.

\section{$2.2 \quad a_{5} \nearrow a_{4}$}

Let us now investigate the coalescence $a_{5} \nearrow a_{4}$. We shall start with the analysis of the Killing vector field $\partial_{t}$ on the set $\left\{\rho=0, z \leq a_{1}\right\}$. A MATHEMATiCA calculation shows that $g_{t t}$ is a rational function with the denominator given by

$$
\left(2\left(a_{3}-a_{1}\right)\left(a_{2}-a_{4}\right)+\left(a_{4}-a_{1}\right) c_{1} c_{2}\right)^{2}\left(z-a_{1}\right)\left(z-a_{2}\right)\left(z-a_{4}\right),
$$

which vanishes as $z$ approaches $a_{1}$ from below. On the other hand, its numerator has the following limit as $z \nearrow a_{1}$,

$$
\left(a_{2}-a_{1}\right)^{2}\left(a_{3}-a_{1}\right)\left(a_{4}-a_{1}\right)^{2}\left(2\left(a_{3}-a_{1}\right)-c_{1}^{2}\right) c_{2}^{2} .
$$

Hence, we have now two possibilities of tuning the parameters to avoid a naked singularity at $\rho=0, z=a_{1}$ :

$$
\begin{aligned}
& \text { 1. } c_{1}= \pm \sqrt{2\left(a_{3}-a_{1}\right)}, \\
& \text { 2. } c_{2}=0 .
\end{aligned}
$$

Keeping them in mind, we shall investigate the behaviour of the Killing vector field $\partial_{t}$ on the set $\left\{\rho=0, a_{4} \leq z \leq a_{3}\right\}$. The function $g_{t t}$ on this domain is a rational function with the denominator

$$
2\left(a_{1}-a_{2}\right)^{2}\left(z-a_{1}\right)\left(z-a_{2}\right)\left(z-a_{4}\right),
$$

vanishing at $z=a_{4}$. On the other hand, the numerator of $g_{t t}$ at $\rho=0, z=a_{4}$ reads

$$
\left(a_{1}-a_{4}\right)^{2}\left(a_{2}-a_{4}\right)^{2}\left(c_{1}-c_{2}\right)^{2} .
$$

Thus, there is only one possibility to avoid a naked singularity at $z=a_{4}$ : set $c_{1}=c_{2}$. Combining the results obtained so far we end up with the following possible fine tunings:

$$
\begin{aligned}
& \text { 1. } c_{1}=c_{2}= \pm \sqrt{2\left(a_{3}-a_{1}\right)}, \\
& \text { 2. } c_{1}=c_{2}=0 .
\end{aligned}
$$

The choice $c_{1}=c_{2}=0$ would bring us back to the seed solution [1], which is nakedly singular, so we are forced to set $c_{1}=c_{2}= \pm \sqrt{2\left(a_{3}-a_{1}\right)}$.

Let us now analyse the behaviour of the Killing vector field $\partial_{\psi}$ on the set $\{\rho=0$, $\left.a_{1} \leq z \leq a_{4}\right\}$. A MathematicA calculation shows that $g_{\psi \psi}$ is a rational function with the denominator given by

$$
-2\left(\left(a_{2}-a_{4}\right) c_{1}+\left(a_{4}-a_{1}\right) c_{2}\right)^{2}\left(z-a_{1}\right)\left(z-a_{2}\right)\left(z-a_{4}\right) .
$$


The singularity at $z=a_{1}$ is cancelled by the tuning (2.3) since the numerator of $g_{\psi \psi}$ at $z=a_{1}$ reads

$$
-\left(a_{2}-a_{1}\right)^{2}\left(a_{4}-a_{1}\right)^{2}\left(2\left(a_{3}-a_{1}\right)-c_{1}^{2}\right)\left(2\left(a_{2}-a_{4}\right)+c_{2} q\right)^{2} .
$$

On the other hand, the denominator of $g_{\psi \psi}$ is singular at $z=a_{4}$ and the numerator has the following limit for $z \nearrow a_{4}$,

$$
\begin{gathered}
2\left(a_{4}-a_{1}\right)^{2}\left(a_{2}-a_{4}\right)^{2}\left(a_{3}-a_{4}\right)\left(2\left(a_{2}-a_{1}\right)-\left(c_{1}-c_{2}\right) q\right)^{2} \\
=8\left(a_{4}-a_{1}\right)^{2}\left(a_{2}-a_{4}\right)^{2}\left(a_{3}-a_{4}\right)\left(a_{2}-a_{1}\right)^{2}
\end{gathered}
$$

which does not vanish. This means that the naked singularity at $\rho=0, z=a_{4}$ persists regardless of the fine tuning of parameters.

We have so far dealt with the situation of the parameters $c_{1}$ and $c_{2}$ assuming finite values. Let us now turn to the case $c_{1} \rightarrow \pm \infty$. In this instance $g_{t t}$, being the norm of the Killing vector $\partial_{t}$, is given in the region $\left\{\rho=0, z \leq a_{1}\right\}$ by the following formula,

$$
-\frac{\left(a_{2}-z\right)\left(a_{3}-z\right)}{\left(a_{1}-z\right)\left(a_{4}-z\right)}
$$

This expression diverges as $z \nearrow a_{1}$ and the singularity cannot be cancelled by any finetuning of the free parameters.

For $c_{2} \rightarrow \pm \infty$ we obtain that $g_{t t}$ on the set $\left\{\rho=0, a_{1} \leq z \leq a_{4}\right\}$ is a rational function with the denominator,

$$
2\left(a_{4}-a_{1}\right)^{2}\left(a_{2}-z\right)\left(a_{4}-z\right)\left(z-a_{1}\right),
$$

vanishing at $z=a_{4}$. On the other hand, its numerator has the following limit for $z \nearrow a_{4}$,

$$
2\left(a_{4}-a_{1}\right)^{2}\left(a_{2}-a_{4}\right)^{2}\left(a_{3}-a_{4}\right) .
$$

We conclude, that in this configuration there is a naked singularity at $\rho=0, z=a_{4}$ that cannot be avoided.

For $c_{1}, c_{2} \rightarrow \pm \infty$ we have $g_{t t}=-\frac{\mu_{2} \mu_{3}}{\mu_{1} \mu_{4}}$, which is singular on the axis $\{\rho=0\}$ in the region $a_{1} \leq z \leq a_{2}$.

\section{$2.3 \quad a_{3} \nearrow a_{2}$}

Let us now consider the coalescence $a_{3} \nearrow a_{2}$.

To rule out smooth non-trivial solutions it is sufficient to investigate the behaviour of the Killing vector field $\partial_{t}$ in the region $\left\{\rho=0, a_{4} \leq z \leq a_{2}\right\}$. With the help of Mathematica we obtain that $g_{t t}$ is a rational function with the denominator given by

$$
2\left(a_{2}-a_{1}\right)^{2}\left(z-a_{1}\right)\left(a_{2}-z\right)\left(a_{5}-z\right),
$$

which vanishes as $z$ approaches $a_{2}$ from below. On the other hand, its numerator has the following limit as $z \nearrow a_{2}$,

$$
-\left(a_{2}-a_{1}\right)^{2}\left(a_{2}-a_{5}\right)^{2} c_{2}^{2}
$$


This means, that one should impose the condition $c_{2}=0$ to avoid a naked singularity at $z=a_{2}$. But setting $a_{3}=a_{2}$ and $c_{2}=0$ completely removes the $S^{3}$ black hole component $[1$, section A.2] and we are left with a single $\psi$-spinning black ring.

In the case $c_{1} \rightarrow \pm \infty$ the function $g_{t t}$ in the region $\left\{\rho=0, z \leq a_{1}\right\}$ reads,

$$
-\frac{\left(a_{2}-z\right)^{2}}{\left(a_{1}-z\right)\left(a_{4}-z\right)} \text {. }
$$

Thus, a naked singularity pops out at $z=a_{1}$.

For $c_{2} \rightarrow \pm \infty, g_{t t}$ in the region $\left\{\rho=0, a_{5} \leq z \leq a_{4}\right\}$ turns out to also be given by,

$$
-\frac{\left(a_{2}-z\right)^{2}}{\left(a_{1}-z\right)\left(a_{4}-z\right)}
$$

now leading to a singularity at $z=a_{4}$.

Similarly to the case described in section 2.2 , for $c_{1}, c_{2} \rightarrow \pm \infty$ we have $g_{t t}=-\frac{\mu_{2}^{2}}{\mu_{1} \mu_{4}}$, which becomes singular on the axis $\{\rho=0\}$ in the whole region $a_{1} \leq z \leq a_{2}$.

\section{$2.4 a_{1} \nearrow a_{5} \nearrow a_{4}$}

According to [1, section A.1] in this limit the Black Saturn metric reduces to a Myers-Perry black hole with a single angular momentum, hence no further analysis is needed. Let us stress however, that to obtain this result independently of the order of the limits one needs to compute the limits at the level of the metric functions - before the imposition of the balance conditions.

\section{$\mathbf{2 . 5} a_{1} \nearrow a_{5}, a_{3} \nearrow a_{2}$}

Let us first investigate the behaviour of the Killing vector field $\partial_{t}$ on the set $\{\rho=0$, $\left.a_{4} \leq z \leq a_{2}\right\}$. Again with the help of MathematicA we obtain the following formula for $g_{t t}$ function in this region

$$
\frac{z-a_{4}}{a_{1}-z}+\frac{c_{2}^{2}}{2\left(a_{2}-z\right)}
$$

We have a naked singularity at $z=a_{2}$ unless we set $c_{2}=0$. As argued in section 2.3 this completely removes the $S^{3}$ black hole component. What is more, the conical singularity detected in section 2.1 persists. Indeed, we have

$$
\lim _{\rho \rightarrow 0} \frac{\rho^{2} g_{\rho \rho}}{g_{\varphi \varphi}}= \begin{cases}k^{2}, & \text { for } z<a_{1} \\ k^{2} \frac{a_{2}-a_{4}}{a_{2}-a_{1}}, & \text { for } a_{4}<z<a_{2} .\end{cases}
$$

Hence, to guarantee the correct periodicity of $\varphi$ we would have to set $a_{4}=a_{1}$, which is excluded by the assumptions of this section.

Since the parameter $c_{1}$ has dropped out of the line element in the coalescence considered in this subsection, we need only to comment on the instance $c_{2} \rightarrow \pm \infty$. In this case, the function $g_{t t}$ in the region $\left\{z \leq a_{1}\right\}$ behaves near the axis $\{\rho=0\}$ like

$$
\frac{4\left(a_{1}-z\right)\left(a_{2}-z\right)^{2}}{\left(a_{4}-z\right) \rho^{2}}+\mathcal{O}\left(\rho^{0}\right)
$$

This excludes the possibility of $c_{2} \rightarrow \pm \infty$ leading to a well-behaved space-time. 
It is sufficient to analyse the behaviour of the Killing vector field $\partial_{t}$ on the axis. In the region $\left\{\rho=0, z \leq a_{1}\right\} g_{t t}$ is a rational function with the denominator given by

$$
\left(2\left(a_{2}-a_{1}\right)\left(a_{2}-a_{4}\right)+\left(a_{4}-a_{1}\right) c_{1} c_{2}\right)^{2}\left(z-a_{1}\right)\left(z-a_{2}\right)\left(z-a_{4}\right) .
$$

As $z \nearrow a_{1}$ its numerator reads

$$
\left(a_{2}-a_{1}\right)^{3}\left(a_{4}-a_{1}\right)^{2}\left(2\left(a_{2}-a_{1}\right)-c_{1}^{2}\right) c_{2}^{2} .
$$

Thus, to avoid a naked singularity at $\rho=0, z=a_{1}$ one has to set

$$
c_{1}= \pm \sqrt{2\left(a_{2}-a_{1}\right)} \quad \text { or } \quad c_{2}=0 .
$$

Let us now switch to the region $\left\{\rho=0, a_{1} \leq z \leq a_{4}\right\}$. A Mathematica calculation shows that $g_{t t}$ is a rational function with the denominator equal to

$$
2\left(\left(a_{2}-a_{4}\right) c_{1}+\left(a_{4}-a_{1}\right) c_{2}\right)^{2}\left(z-a_{1}\right)\left(z-a_{2}\right)\left(z-a_{4}\right) .
$$

The continuity of $g_{t t}$ at $z=a_{1}$ is easily verified for both choices of parameters (2.5). On the other hand, as $z$ approaches $a_{4}, g_{t t}$ becomes singular since its numerator at $z=a_{4}$ reads

$$
2\left(a_{1}-a_{4}\right)^{2}\left(a_{2}-a_{4}\right)^{3}\left(c_{1}-c_{2}\right)^{2} .
$$

To bypass the naked singularity at $\rho=0, z=a_{4}$ we need to set $c_{1}=c_{2}$ in addition to (2.5).

Finally, in the region $\left\{\rho=0, a_{4} \leq z \leq a_{2}\right\}$ the denominator of $g_{t t}$ is given by

$$
2\left(a_{1}-a_{2}\right)^{2}\left(z-a_{1}\right)\left(z-a_{2}\right)\left(z-a_{4}\right) .
$$

Again, the continuity of $g_{t t}$ at $z=a_{4}$ is guaranteed by the tuning of parameters imposed so far. However, the numerator of $g_{t t}$ at $z=a_{2}$ reads

$$
-\left(a_{1}-a_{2}\right)^{2}\left(a_{2}-a_{4}\right)^{2} c_{2}^{2},
$$

so the only way to avoid a singularity at $z=a_{2}$ is to set $c_{2}=0$. Combining this with the previous results we conclude that to assure the smoothness of the Killing vector field $\partial_{t}$ on the axis $\{\rho=0\}$ one needs to set $c_{1}=c_{2}=0$. As already argued, this would bring us back to the seed solution [1], which is singular itself.

It remains to check the possibility of cancelling the singularities by letting one or both of the parameters $c_{1}, c_{2}$ go to $\pm \infty$. As $c_{1} \rightarrow \pm \infty$ we obtain that $g_{t t}$ in the region $\left\{\rho=0, z \leq a_{1}\right\}$ is given by the expression

$$
-\frac{\left(a_{2}-z\right)^{2}}{\left(a_{1}-z\right)\left(a_{4}-z\right)}
$$

singular at $z=a_{1}$. 
For $c_{2} \rightarrow \pm \infty$ on the other hand, we obtain the following behaviour of $g_{t t}$ near the axis $\{\rho=0\}$ in the region $\left\{a_{4} \leq z \leq a_{2}\right\}$,

$$
\frac{4\left(a_{2}-z\right)^{2}\left(a_{4}-z\right)}{\left(a_{1}-z\right) \rho^{2}}+\mathcal{O}\left(\rho^{0}\right) .
$$

Moreover, if we let both $c_{1}$ and $c_{2}$ tend to infinity we again obtain $g_{t t}=-\frac{\mu_{2}^{2}}{\mu_{1} \mu_{4}}$.

We conclude that the Black Saturn solution with $a_{5} \nearrow a_{4}, a_{3} \nearrow a_{2}$ and one or both of the $c_{i}$ parameters infinite is nakedly singular.

$\mathbf{2 . 7} a_{1} \nearrow a_{5} \nearrow a_{4}, a_{3} \nearrow a_{2}$

As in the previous cases (see section 2.1) the limit $a_{1} \nearrow a_{5}$ implies that the parameter $c_{1}$ is no longer present in the line element. Furthermore, an investigation of the behaviour of the Killing vector $\partial_{t}$ on the axis forces us to impose $c_{2}=0$. Indeed, in the region $\left\{\rho=0, a_{1} \leq z \leq a_{2}\right\}$ the metric function $g_{t t}$ reads

$$
\frac{2\left(z-a_{2}\right)+c_{2}^{2}}{2\left(a_{2}-z\right)}
$$

so only $c_{2}=0$ allows to avoid a singularity at $z=a_{2}$. But if $c_{1}$ drops out of the metric functions and $c_{2}$ vanishes we are again back at the seed solution [1], which is of no physical interest.

Moreover, in the case $c_{2} \rightarrow \pm \infty$ we obtain $g_{t t}=\frac{\mu_{2}^{2}}{\rho^{2}}$, that clearly leads to singularities on the axis.

\section{Conclusions}

We have investigated various different coalescences of parameters defining the Black Saturn solution. We have shown that either the resulting metric is nakedly singular or it reduces to a black hole with one connected component of the event horizon: a Myers-Perry black hole or Emperano-Reall black ring.

Led by the example given by Geroch in [12] one might think that there can still be a way of obtaining a meaningful coalescence limit in the Black Saturn family by employing a smart change of coordinate chart. However, as demonstrated in [12], the Killing vectors are inherited by any limit of a space-time with some parameters. Strictly speaking, this property has been demonstrated for a 3+1-dimensional case. Nevertheless, as the technique developed in [12, appendix B] is general, the proof can be adapted in a straightforward way to a $4+1$ dimensional space-time with three Killing vectors. Now, since our analysis consisted in uncovering singularities in the norms of Killing vector fields, we conclude that any coordinate transformation would either lead to the same results or not yield a proper limit space-time at all.

We have thus exhausted the possibility of constructing a smooth extremal Black Saturn configuration in the family of solutions of Elvang-Figueras.

This outcome is in consent with the known properties of 4+1-dimensional black holes. Both spherical black holes [2] and black rings [13, 14] require two non-vanishing angular 
momenta to admit smooth extremal configurations. Unfortunately, the Black Saturn solution of Elvang-Figueras has angular momentum in a single plane only and it is not clear if doubly-spinning components can at all be kept in balance [1]. Thus, the question of the existence of smooth stationary axisymmetric black holes with disconnected degenerate Killing horizons in $4+1$ dimensions remains open.

\section{Acknowledgments}

We would like to thank Piotr T. Chruściel for suggesting this problem to us and for his illuminating remarks. We also thank Patryk Mach and Sebastian Szybka for comments on the manuscript.

The main part of the calculations was carried out using Mathematica 8.0.4 by Wolfram Research.

Project operated within the Foundation for Polish Science IPP Programme "Geometry and Topology in Physical Models" co-financed by the EU European Regional Development Fund, Operational Program Innovative Economy 2007-2013. Partial support of the Polish Ministry of Science and Higher Education under the grant for young scientists and $\mathrm{PhD}$ students is acknowledged.

\section{A The Black Saturn metric}

In the generalised Weyl coordinates $(t, \rho, z, \psi, \varphi)$ the Black Saturn line element [1] reads

$$
\begin{aligned}
d s^{2} & =g_{t t} d t^{2}+g_{t \psi} d t d \psi+g_{\psi \psi} d \psi^{2}+g_{\rho \rho} d \rho^{2}+g_{z z} d z^{2}+g_{\varphi \varphi} d \varphi^{2} \\
& =-\frac{H_{y}}{H_{x}}\left[d t+\left(\frac{\omega_{\psi}}{H_{y}}+q\right) d \psi\right]^{2}+H_{x}\left\{k^{2} P\left(d \rho^{2}+d z^{2}\right)+\frac{G_{y}}{H_{y}} d \psi^{2}+\frac{G_{x}}{H_{x}} d \varphi^{2}\right\},
\end{aligned}
$$

where $k, q$ are real constants. The metric functions depend only on variables $\rho$ and $z$. Define

$$
\mu_{i}:=\sqrt{\rho^{2}+\left(z-a_{i}\right)^{2}}-\left(z-a_{i}\right),
$$

where the $a_{i}$ 's are real constants. The assumed ordering of $a_{i}$ 's (1.1) implies

$$
\mu_{1} \leq \mu_{5} \leq \mu_{4} \leq \mu_{3} \leq \mu_{2} \quad \text { and } \quad \mu_{i}=\mu_{j} \Leftrightarrow a_{i}=a_{j}
$$

Let us list the functions constituting the line element (A.1):

$$
\begin{aligned}
G_{x} & =\frac{\rho^{2} \mu_{4}}{\mu_{3} \mu_{5}} \\
P & =\left(\mu_{3} \mu_{4}+\rho^{2}\right)^{2}\left(\mu_{1} \mu_{5}+\rho^{2}\right)\left(\mu_{4} \mu_{5}+\rho^{2}\right), \\
H_{x} & =F^{-1}\left[M_{0}+c_{1}^{2} M_{1}+c_{2}^{2} M_{2}+c_{1} c_{2} M_{3}+c_{1}^{2} c_{2}^{2} M_{4}\right], \\
H_{y} & =F^{-1} \frac{\mu_{3}}{\mu_{4}}\left[M_{0} \frac{\mu_{1}}{\mu_{2}}-c_{1}^{2} M_{1} \frac{\rho^{2}}{\mu_{1} \mu_{2}}-c_{2}^{2} M_{2} \frac{\mu_{1} \mu_{2}}{\rho^{2}}+c_{1} c_{2} M_{3}+c_{1}^{2} c_{2}^{2} M_{4} \frac{\mu_{2}}{\mu_{1}}\right],
\end{aligned}
$$


where $c_{1}$ and $c_{2}$ are real constants, and

$$
\begin{aligned}
M_{0}= & \mu_{2} \mu_{5}^{2}\left(\mu_{1}-\mu_{3}\right)^{2}\left(\mu_{2}-\mu_{4}\right)^{2}\left(\rho^{2}+\mu_{1} \mu_{2}\right)^{2}\left(\rho^{2}+\mu_{1} \mu_{4}\right)^{2}\left(\rho^{2}+\mu_{2} \mu_{3}\right)^{2}, \\
M_{1}= & \mu_{1}^{2} \mu_{2} \mu_{3} \mu_{4} \mu_{5} \rho^{2}\left(\mu_{1}-\mu_{2}\right)^{2}\left(\mu_{2}-\mu_{4}\right)^{2}\left(\mu_{1}-\mu_{5}\right)^{2}\left(\rho^{2}+\mu_{2} \mu_{3}\right)^{2}, \\
M_{2}= & \mu_{2} \mu_{3} \mu_{4} \mu_{5} \rho^{2}\left(\mu_{1}-\mu_{2}\right)^{2}\left(\mu_{1}-\mu_{3}\right)^{2}\left(\rho^{2}+\mu_{1} \mu_{4}\right)^{2}\left(\rho^{2}+\mu_{2} \mu_{5}\right)^{2}, \\
M_{3}= & 2 \mu_{1} \mu_{2} \mu_{3} \mu_{4} \mu_{5}\left(\mu_{1}-\mu_{3}\right)\left(\mu_{1}-\mu_{5}\right)\left(\mu_{2}-\mu_{4}\right)\left(\rho^{2}+\mu_{1}^{2}\right)\left(\rho^{2}+\mu_{2}^{2}\right) \\
& \times\left(\rho^{2}+\mu_{1} \mu_{4}\right)\left(\rho^{2}+\mu_{2} \mu_{3}\right)\left(\rho^{2}+\mu_{2} \mu_{5}\right), \\
M_{4}= & \mu_{1}^{2} \mu_{2} \mu_{3}^{2} \mu_{4}^{2}\left(\mu_{1}-\mu_{5}\right)^{2}\left(\rho^{2}+\mu_{1} \mu_{2}\right)^{2}\left(\rho^{2}+\mu_{2} \mu_{5}\right)^{2}
\end{aligned}
$$

and

$$
\begin{aligned}
F= & \mu_{1} \mu_{5}\left(\mu_{1}-\mu_{3}\right)^{2}\left(\mu_{2}-\mu_{4}\right)^{2}\left(\rho^{2}+\mu_{1} \mu_{3}\right)\left(\rho^{2}+\mu_{2} \mu_{3}\right)\left(\rho^{2}+\mu_{1} \mu_{4}\right) \\
& \times\left(\rho^{2}+\mu_{2} \mu_{4}\right)\left(\rho^{2}+\mu_{2} \mu_{5}\right)\left(\rho^{2}+\mu_{3} \mu_{5}\right) \prod_{i=1}^{5}\left(\rho^{2}+\mu_{i}^{2}\right) .
\end{aligned}
$$

Furthermore,

$$
G_{y}=\frac{\mu_{3} \mu_{5}}{\mu_{4}}
$$

and the off-diagonal part of the metric is governed by

$$
\omega_{\psi}=2 \frac{c_{1} R_{1} \sqrt{M_{0} M_{1}}-c_{2} R_{2} \sqrt{M_{0} M_{2}}+c_{1}^{2} c_{2} R_{2} \sqrt{M_{1} M_{4}}-c_{1} c_{2}^{2} R_{1} \sqrt{M_{2} M_{4}}}{F \sqrt{G_{x}}},
$$

with $R_{i}=\sqrt{\rho^{2}+\left(z-a_{i}\right)^{2}}$.

The determinant of the metric reads

$$
\operatorname{det} g_{\mu_{\nu}}=-\rho^{2} H_{x}^{2} k^{4} P^{2} .
$$

Open Access. This article is distributed under the terms of the Creative Commons Attribution License which permits any use, distribution and reproduction in any medium, provided the original author(s) and source are credited.

\section{References}

[1] H. Elvang and P. Figueras, Black Saturn, JHEP 05 (2007) 050 [hep-th/0701035] [InSPIRE].

[2] R.C. Myers and M.J. Perry, Black holes in higher dimensional space-times, Annals Phys. 172 (1986) 304 [INSPIRE].

[3] R. Emparan and H.S. Reall, A rotating black ring solution in five-dimensions, Phys. Rev. Lett. 88 (2002) 101101 [hep-th/0110260] [INSPIRE].

[4] A.A. Pomeransky and R.A. Sen'kov, Black ring with two angular momenta, hep-th/0612005 [INSPIRE].

[5] P.T. Chruściel, M. Eckstein, L. Nguyen and S.J. Szybka, Existence of singularities in two-Kerr black holes, Class. Quant. Grav. 28 (2011) 245017 [arXiv:1111.1448] [INSPIRE].

[6] J. Hennig and G. Neugebauer, Non-existence of stationary two-black-hole configurations: the degenerate case, Gen. Rel. Grav. 43 (2011) 3139 [arXiv:1103.5248] [INSPIRE]. 
[7] G. Neugebauer and J. Hennig, Non-existence of stationary two-black-hole configurations, Gen. Rel. Grav. 41 (2009) 2113 [arXiv:0905.4179] [INSPIRE].

[8] G. Neugebauer and J. Hennig, Stationary two-black-hole configurations: a non-existence proof, J. Geom. Phys. 62 (2012) 613 [arXiv:1105.5830] [INSPIRE].

[9] V. Belinski and E. Verdaguer, Gravitational solitons, Cambridge University Press, Cambridge U.K. (2001) [INSPIRE].

[10] P.T. Chruściel, M. Eckstein and S.J. Szybka, On smoothness of Black Saturns, JHEP 11 (2010) 048 [arXiv: 1007.3668] [InSPIRE].

[11] S.J. Szybka, Stable causality of Black Saturns, JHEP 05 (2011) 052 [arXiv: 1102.3942] [INSPIRE].

[12] R.P. Geroch, Limits of spacetimes, Commun. Math. Phys. 13 (1969) 180 [inSPIRE].

[13] P.T. Chruściel, J. Cortier and A.G.-P. Gómez-Lobo, On the global structure of the Pomeransky-Senkov black holes, Adv. Theor. Math. Phys. 14 (2010) 1779 [arXiv:0911.0802] [INSPIRE].

[14] P. Figueras, H.K. Kunduri, J. Lucietti and M. Rangamani, Extremal vacuum black holes in higher dimensions, Phys. Rev. D 78 (2008) 044042 [arXiv: 0803.2998] [INSPIRE]. 